\title{
EDITORIAL
}

\section{CRISPR innovations in plant breeding}

\author{
Sang-Gyu Kim ${ }^{1}$
}

Received: 22 April 2021 / Accepted: 23 April 2021 / Published online: 2 May 2021

(c) The Author(s), under exclusive licence to Springer-Verlag GmbH Germany, part of Springer Nature 2021

Ten years have passed since Emmanuelle Charpentier and Jennifer Doudna reported the mechanism underlying a bacterial immune system, CRISPR (clustered regularly interspaced short palindromic repeats), which can be engineered to induce highly accurate, targeted mutations in any genome (Jinek et al. 2012). In the meantime, CRISPR innovations make it possible for plant molecular biologists and crop breeders to collaborate to improve crop productivity. For instance, improved soybean with high oleic acid and low linoleic and linolenic acids was generated by removing several nucleotides in two fatty acid desaturase genes (Haun et al. 2014); the oils extracted from the edited soybean are now commercially available (Calyno ${ }^{\mathrm{TM}}$, Calyxt). CRISPRmediated base-editing has been used to produce herbicideresistant crops with no transgenes present crop genomes (Kang et al. 2019; Li et al. 2020). Plant structure (Xu et al. 2016), fruit size, and nutrient content (Lemmon et al. 2018) have been improved via CRISPR.

In this special issue, we look back on the development of plant-genome-editing tools over the past 10 years and look into the future of crop genome-editing innovations that are made possible by diverse CRISPR systems. Alvarez et al. (2021) discussed the regulation issues with gene-edited crops. Naturally occurring mutations and edits of small insertion and deletion mutations or substitution mutations may be indistinguishable in crops, which may spur changes in regulations, as we have seen in the United States and elsewhere (Alvarez et al. 2021). Plant virus has been modified to become both the cargo and the vehicle that delivers CRISPR components into plant cells. The most widely used CRISPR system consists of one large protein, an engineered endonuclease (i.e. Cas9), and a short single-strand guide RNA to guide the nuclease to the target site. Plant DNA or RNA viruses may act as efficient vectors to CRISPR reagents into

Sang-Gyu Kim

sgkim1@kaist.ac.kr

1 Department of Biological Sciences, Korea Advanced Institute for Science and Technology, Daejeon 34141, Republic of Korea plant cells, including germ cells to generate edited progeny without plant regeneration. Kujur et al. (2021) summarized the development of a virus-induced gene-editing system and the current limitation of this system. Miladinović et al. (2021) reviewed how the CRISPR technology serves as a bridge from lab-based molecular biology to field-based crop breeding, describing in detail the traits of crops that have been targeted over the last decade. Huang et al. (2021) focused on how genome-editing technology can be used to change plant architecture that improves crop productivity. Biswas et al. (2021) discussed future directions of precise crop breeding; CRISPR systems that have been advanced to regulate haploid induction and to maintain heterosis in crops.

This special issue also covers innovations of genomeediting in various crops: tomato, rice, petunia, and soybean. Tran et al. (2021) precisely removed the domain of the target protein, which enhanced stress tolerance in tomato. Jung et al. (2021a) showed that the photosynthetic ability of rice can be manipulated by genome-editing. Pharmaceutical protein production in plants can be complicated by glycosylation patterns and Jung et al. (2021b) effectively altered glycosylation patterns of proteins in rice using CRISPR. Yu et al. (2021) innovated a method to edit petunia by delivering ribonucleoprotein into protoplasts to regenerate the whole plants from edited cells. Nagy et al. (2021) attempted to create a new combination of the NBS-LRR family that exists within the soybean genome in tandem-repeat form. The editing efficiency of a CRISPR system, which is mainly determined by the guide RNA, is especially examined in transformation-recalcitrant species such as soybean. Thus, Kim and Choi (2020) developed a method to examine guide RNA efficiency in soybean protoplasts prior to undertaking lengthy and laborious stable transformation.

CRISPR-based precision crop breeding is in its infancy. Incremental and revolutionary discoveries alike are in our view as we gaze into the future. By continuing research progress based on the foundation of innovations in this special issue of Plant Cell Reports, we can expect real improvements in agricultural production and sustainability. By 
understanding the molecular mechanisms of agronomic traits and maintaining genetic diversity of crops, the power of genome-editing tools for crop improvement can be unleashed.

\section{References}

Alvarez D et al (2021) Fruit crops in the era of genome editing-closing the regulatory gap. Plant Cell Rep. https://doi.org/10.1007/ s00299-021-02664-X

Biswas S et al (2021) CRISPR/Cas systems: opportunities and challenges for crop breeding. Plant Cell Rep. https://doi.org/10.1007/ s00299-021-02708-2

Haun W, Coffman A, Clasen BM et al (2014) Improved soybean oil quality by targeted mutagenesis of the fatty acid desaturase 2 gene family. Plant Biotechnol J 12:934-940

Huang X et al (2021) Modification of cereal plant architecture by genome editing to improve yields. Plant Cell Rep. https://doi.org/ 10.1007/s00299-021-02668-7

Jinek M, Chylinski K, Fonfara I et al (2012) A programmable dualRNA-guided DNA endonuclease in adaptive bacterial immunity. Science 337:816-821

Jung YJ et al (2021a) Transcriptomic and physiological analysis of OsCAO1 knockout lines using the CRISPR/Cas9 system in rice. Plant Cell Rep. https://doi.org/10.1007/s00299-020-02607-y

Jung JW et al (2021b) Inactivation of the $\beta(1,2)$-xylosyltransferase and the $\alpha(1,3)$-fucosyltransferase gene in rice (Oryza sativa) by multiplex CRISPR/Cas9 strategy. Plant Cell Rep. https://doi.org/ 10.1007/s00299-021-02667-8

Kang BC, Woo JW, Kim ST et al (2019) Guidelines for C to T base editing in plants: base-editing window, guide RNA length, and efficient promoter. Plant Biotechnol Rep 13:533-541
Kim H, Choi J (2020) A robust and practical CRISPR/crRNA screening system for soybean cultivar editing using LbCpf1 ribonucleoproteins. Plant Cell Rep. https://doi.org/10.1007/s00299-020-02597-x

Kujur S et al (2021) Plant viral vectors: expanding the possibilities of precise gene editing in plant genomes. Plant Cell Rep. https://doi. org/10.1007/s00299-021-02697-2

Lemmon ZH, Reem NT, Dalrymple J et al (2018) Rapid improvement of domestication traits in an orphan crop by genome editing. Nat Plants 4:766-770

Li C, Zhang R, Meng X et al (2020) Targeted, random mutagenesis of plant genes with dual cytosine and adenine base editors. Nat Biotechnol 38:875-882

Miladinović S et al (2021) Targeted plant improvement through genome editing: from lab to field. Plant Cell Rep. https://doi.org/ 10.1007/s00299-020-02655-4

Nagy ED et al (2021) Novel disease resistance gene paralogs created by CRISPR/Cas9 in soy. Plant Cell Rep. https://doi.org/10.1007/ s00299-021-02678-5

Tran MT et al (2021) CRISPR/Cas9-based precise excision of SlHyPRP1 domain(s) to obtain salt stress- tolerant tomato. Plant Cell Rep. https://doi.org/10.1007/s00299-020-02622-z

Xu C, Park SJ, Van Eck J, Lippman ZB (2016) Control of inflorescence architecture in tomato by $\mathrm{BTB} / \mathrm{POZ}$ transcriptional regulators. Genes Develop 30:2048-2061

Yu J et al (2021) Simultaneous targeting of duplicated genes in Petunia protoplasts for flower color modification via CRISPR-Cas9 ribonucleoproteins. Plant Cell Rep. https://doi.org/10.1007/ s00299-020-02593-1

Publisher's Note Springer Nature remains neutral with regard to jurisdictional claims in published maps and institutional affiliations. 